\title{
Stigmatization from Work-Related COVID-19 Exposure: A Systematic Review with Meta-Analysis
}

\author{
Melanie Schubert ${ }^{1, * \mathbb{C}}$, Julia Ludwig ${ }^{2}$, Alice Freiberg ${ }^{1}$, Taurai Monalisa Hahne ${ }^{3} \mathbb{D}$, Karla Romero Starke ${ }^{1,4} \mathbb{D}^{\mathbb{D}}$, \\ Maria Girbig ${ }^{1}{ }^{\infty}$, Gudrun Faller ${ }^{5}$, Christian Apfelbacher ${ }^{3}{ }^{\infty}$, Olaf von dem Knesebeck ${ }^{2}$ and Andreas Seidler ${ }^{1}{ }^{(D)}$ \\ 1 Institute and Policlinic of Occupational and Social Medicine, Faculty of Medicine, \\ Technische Universität Dresden, 01307 Dresden, Germany; alice.freiberg@tu-dresden.de (A.F.); \\ karla.romero_starke@tu-dresden.de (K.R.S.); maria.girbig@tu-dresden.de (M.G.); \\ andreas.seidler@tu-dresden.de (A.S.) \\ 2 Institute of Medical Sociology, University Medical Center Hamburg-Eppendorf, 20246 Hamburg, Germany; \\ j.ludwig@uke.de (J.L.); o.knesebeck@uke.de (O.v.d.K.) \\ 3 Institute of Social Medicine and Health Systems Research, Otto von Guericke University Magdeburg, \\ 39120 Magdeburg, Germany; taurai.hahne@med.ovgu.de (T.M.H.); \\ christian.apfelbacher@med.ovgu.de (C.A.) \\ 4 Institute of Sociology, Faculty of Behavioural and Social Sciences, Chemnitz University of Technology, \\ Thüringer Weg 9, 09126 Chemnitz, Germany \\ 5 Department of Community Health, Hochschule für Gesundheit, 44801 Bochum, Germany; \\ gudrun.faller@hs-gesundheit.de \\ * Correspondence: melanie.schubert@tu-dresden.de
}

\section{check for} updates

Citation: Schubert, M.; Ludwig, J.; Freiberg, A.; Hahne, T.M.; Romero Starke, K.; Girbig, M.; Faller, G.; Apfelbacher, C.; von dem Knesebeck, O.; Seidler, A. Stigmatization from Work-Related COVID-19 Exposure: A Systematic Review with Meta-Analysis. Int. J. Environ. Res. Public Health 2021, 18, 6183. https:// doi.org/10.3390/ijerph18126183

Academic Editor: Luenda E. Charles

Received: 10 May 2021

Accepted: 3 June 2021

Published: 8 June 2021

Publisher's Note: MDPI stays neutral with regard to jurisdictional claims in published maps and institutional affiliations.

Copyright: (c) 2021 by the authors. Licensee MDPI, Basel, Switzerland. This article is an open access article distributed under the terms and conditions of the Creative Commons Attribution (CC BY) license (https:// creativecommons.org/licenses/by/ $4.0 /)$.

\begin{abstract}
Stigmatization from work-related COVID-19 exposure has not been investigated in detail yet. Therefore, we systematically searched three databases: Medline, Embase, and PsychInfo (until October 2020), and performed a grey literature search (until February 2021). We identified 46 suitable articles from 24 quantitative and 11 qualitative studies, 6 systematic reviews, 3 study protocols and 1 intervention. The assessment of stigmatization varied widely, ranging from a single-item question to a 22-item questionnaire. Studies mostly considered perceived self-stigma (27 of 35 original studies) in healthcare workers (HCWs) or hospital-related jobs (29 of 35). All articles reported on stigmatization as a result of work-related COVID-19 exposure. However, most quantitative studies were characterized by convenience sampling (17 of 24), and all studies-also those with an adequate sampling design-were considered of low methodological quality. Therefore, it is not possible to determine prevalence of stigmatization in defined occupational groups. Nevertheless, the workrelated stigmatization of occupational groups with or without suspected contact to COVID-19 is a relevant problem and increases the risk for depression (odds ratio $(\mathrm{OR})=1.74 ; 95 \%$ confidence interval CI 1.29-2.36) and anxiety (OR $=1.75 ; 95 \%$ CI 1.29-2.37). For promoting workers' health, anti-stigma strategies and support should be implemented in the workplace.
\end{abstract}

Keywords: corona; COVID-19; bullying; discrimination; healthcare workers; nursing; SARS-CoV2; stigma; work

\section{Introduction}

The COVID-19 pandemic has had a dramatic impact on all aspects of our lives. Besides the direct effects of the pandemic, there are also many indirect social consequences. People's everyday life is disrupted and negatively affected by the pandemic. Compliance with the hygiene measures and in particular the "infodemic", which is characterized by an overabundance of news covering facts, rumours and misinformation [1], have triggered or strengthened negative feelings such as fear, anger and hatred in the population. International and national media report on a "witch-hunt hysteria", attacks on index cases, infected people and relatives, and stigmatization $[2,3]$. 
The stigmatization process includes labelling a person with a specific characteristic, linking the label to undesirable characteristics (stereotypes) which result in negative emotional reactions. This constitutes the basis for the separation of "us" and "them", and leads to discrimination and the loss of status [4]. Furthermore, the stigmatization process depends on the social, political and economic power of the stigmatized group [4].

On the action-oriented level of stigmatization, a distinction between public stigma, stigma by association and self-stigma can be made [5]. Thereby, endorsed stereotypes, prejudice, and discrimination in the general public against a particular group are defined as public stigma [6]. When this stigmatization process is transferred to relatives of the stigmatized individuals, it is referred to as associative stigma [7]. Self-stigmatization, on the other hand, is defined as the internalization of the very same stereotypes and prejudices [6] and can be additionally divided into perceived (belief that "most" people devalue and discriminate against the individuals holding the specific characteristic) [8] and anticipated stigma (expectation of experiencing prejudice and discrimination based on the characteristic) [9].

The consequences of stigmatization can be diverse for those affected and include discrimination-related stress, reduced self-esteem and reduced self-efficacy [10]. Stigmatization can lead to a reduced quality of life [11], mental illnesses, trauma, and even suicide [12-15]. Furthermore, the stigma may not be disclosed, which is associated with less use of professional help, and poorer assessment of one's own health [16]. As a result, the feared stigmatization by the public may lead to a concealment of the disease $[9,17]$, and may increase the spread of it. In association with work, stigmatized employees are less able to cope with daily work demands [12], and also report lower job satisfaction, job performance, work commitment, and willingness to learn and develop $[18,19]$. Furthermore, stigmatization can also initiate or solidify career-related consequences such as decline [20], dismissals or "voluntary" dismissals [21].

Workers exposed to potential hazards and diseases such as COVID-19 may also suffer from stigmatization. Stigmatization is a key feature of bullying [22,23]. Leymann [22] and Einarsen [24] identified four phases: aggressive behaviour, bullying, stigma and severe trauma. The initial phase is characterized by indirect and direct aggressive behaviour which leaves the affected person humiliated and increasingly isolated. The persons become more and more stigmatized, making it more difficult to for them to defend themselves. Stigmatization makes it less possible for the person concerned to cope with daily work demands [12]. There are also associations with lower job satisfaction, job performance, work commitment, willingness to learn $[18,19]$. Stigmatization can also initiate or consolidate the relegation of careers [20] and dismissals or "voluntary" dismissals [21].

Stigmatization can result in an additional high psychological stress for workers. Recent systematic reviews indicate that stigmatization is a risk factor for mental disorders in healthcare workers (HCWs) caring for MERS-CoV2/SARS-patients [25,26]. Recently, a large number of systematic reviews investigating the psychological impact of work-related COVID-19 exposure such as stigmatization have been published. However, these reviews only include no or very few original studies related to COVID-19 [27-34]. Thus far, a systematic review of the literature on work-related stigmatization focussing on COVID-19 is missing. The aim of this systematic review was to provide a comprehensive overview of COVID-19-related stigmatization across occupational classes. Furthermore, we aimed to summarize health consequences of work-related stigmatization from COVID-19 exposure using a meta-analytic approach.

\section{Materials and Methods}

\subsection{Review Questions and Study Eligibility Criteria}

We systematically searched for publications on the stigmatization experience of employees due to COVID-19. Our main question was whether employees experience stigmatization in association with work due to COVID-19. Related to the main objective, we were interested in describing the specific forms of stigmatization (e.g., public stigma, self-stigma, 
associative stigma), how often they were observed in specific occupational groups, and if employees returning to work (such as after quarantine, or infection) experience stigmatization (A). Furthermore, we aimed to identify health consequences as a result of work-related stigmatization due to COVID-19 (B), and organizational or other conditions that increase or prevent work-related stigmatization in association with COVID-19 (C).

We followed the procedures outlined in our study protocol registered a priori on Open Science Framework (https:/ / osf.io/82zav accessed on 3 June 2021).

According to the population-exposure-outcome (PEO) criteria, we designed our systematic search strategy to include studies of the working population $(\mathrm{P})$. While the search strategy was designed to include all studies on new infectious diseases (including SARSCoV-2, SARS, MERS, influenza virus H1N1, and influenza virus H7N9), this paper focuses on the results of the search on COVID-19 (E). We included all studies on stigmatization of any type (including bullying) in association with work due to COVID-19 assessed with validated or non-validated instruments $(\mathrm{O})$. Furthermore, we included any measures to prevent or deal with occupational stigmatization. Inclusion and exclusion criteria are listed in Table 1.

Table 1. Inclusion and exclusion criteria for the research question concerning work-related stigmatization as outcome (research question A and C).

\begin{tabular}{cll}
\hline Category & \multicolumn{1}{c}{ Inclusion } & \multicolumn{1}{c}{ Exclusion } \\
\hline Population & General working population (all sexes) & $\begin{array}{l}\text { Children and youth, unemployed persons or persons in } \\
\text { non-paid employment, pensioners, and persons over 70 years }\end{array}$ \\
\hline Exposure & SARS-CoV-2 (A) & Other infectious diseases such as HIV and tuberculosis ${ }^{1}$ \\
\hline \multirow{2}{*}{ Outcomes } & $\begin{array}{l}\text { All stigmatization forms (including bullying) in } \\
\text { association with work (A); measures that prevent } \\
\text { work-related stigmatization (C) }\end{array}$ & - \\
\hline
\end{tabular}

${ }^{1}$ Results on new respiratory virus diseases other than COVID-19, i.e., SARS, MERS, influenza virus H1N1, and influenza virus H7N9 were excluded in the further study process.

Additionally, we studied health consequences $(\mathrm{O})$ from the stigmatization of employees (P) due to COVID-19 (E). The PEO for this is shown in Table 2.

Table 2. Inclusion and exclusion criteria for the research question concerning health consequences of stigmatization (research question B).

\begin{tabular}{cll}
\hline Category & \multicolumn{1}{c}{ Inclusion } & \multicolumn{1}{c}{ Exclusion } \\
\hline Population & General working population (all sexes) & $\begin{array}{l}\text { Children and youth, unemployed persons or } \\
\text { persons in non-paid employment, pensioners, and } \\
\text { persons above 70 years }\end{array}$ \\
\hline Exposure & Work-related stigmatization (all forms) due to COVID-19 & - \\
\hline Outcomes & Physical and mental health, stigma-reducing strategies & - \\
\hline
\end{tabular}

We included primary studies (cohort, case-control, case-cohort, RCT, cross-sectional studies, ecological studies, qualitative studies), systematic reviews, dissertations with primary data, or a systematic literature search. Abstracts only, clinical observation studies, books, book chapters, book reviews, comments, corrections, editorials, introductions, forewords, letters, replies, popular science media, and narrative reviews were excluded from this systematic review. Publications with no abstract were included if the title seemed to be relevant to the topic of this review.

We used no geographic or language restrictions, but titles and abstracts not written in English or German were excluded. 


\subsection{Definition of Stigma Forms}

Stigmatization forms were differentiated according to the definition by Pescosolido and Martin [5]. Descriptions and examples of each stigmatization form from the included studies are given in Table 3.

Table 3. Definition of stigma forms of included studies.

\begin{tabular}{|c|c|c|}
\hline Stigma Forms & Description & Examples from Included Studies \\
\hline Public stigma & $\begin{array}{l}\text { Endorsement of stereotypes, prejudices and } \\
\text { discrimination against a group, that holds a } \\
\text { specific characteristic. In surveys, the public } \\
\text { is asked. }\end{array}$ & $\begin{array}{l}\text { Stereotypes: e.g., "Healthcare workers who work in } \\
\text { hospitals are likely to have COVID-19" [35]. } \\
\text { Discrimination: e.g., "Healthcare workers should } \\
\text { have some restrictions on their freedom [35]. } \\
\text { Social exclusion as a component of discrimination: } \\
\text { e.g., "I do not want to be around someone who } \\
\text { works in a healthcare setting [35]. }\end{array}$ \\
\hline Associative stigma & $\begin{array}{l}\text { Endorsement of stereotypes, prejudices and } \\
\text { discrimination against a group which is } \\
\text { transferred to relatives. In surveys, the public or } \\
\text { affected persons are asked. }\end{array}$ & $\begin{array}{l}\text { Discrimination: e.g., "Relatives being alienated } \\
\text { because employment related to COVID-19" [36]. } \\
\text { Social exclusion as a component of discrimination: } \\
\text { e.g., "People would avoid my family members } \\
\text { because of my job" [37]. } \\
\text { Aggressive behavior/bullying as a component of } \\
\text { discrimination: e.g., "Have verbally abused (... ) or } \\
\text { physically assaulted (... ) my family members" [38]. }\end{array}$ \\
\hline $\begin{array}{l}\text { Self-stigma } \\
\text { (internalized) }\end{array}$ & $\begin{array}{l}\text { Internalization, i.e., perception and transfer of } \\
\text { stereotypes and devaluations to the own person. } \\
\text { In surveys, affected persons are asked. }\end{array}$ & $\begin{array}{l}\text { e.g., "Felt guilty about possibly exposing family, } \\
\text { community and peers to infection" [38]; "feeling } \\
\text { inferior to others due to occupation" [39]. }\end{array}$ \\
\hline Self-stigma (perceived) & $\begin{array}{l}\text { Belief that "most people" will devalue and } \\
\text { discriminate the stigmatized. In surveys, affected } \\
\text { persons are asked. }\end{array}$ & $\begin{array}{l}\text { Stereotypes: e.g., "People do not trust me and worry } \\
\text { that I might infect them" [38]. } \\
\text { Discrimination: e.g., "People feel uncomfortable } \\
\text { when I am around" [40] } \\
\text { Social exclusion as a component of discrimination: } \\
\text { e.g., "Family members and friends have avoided } \\
\text { contact with me because of my work?" [41]. } \\
\text { Aggressive behavior/bullying as a component of } \\
\text { discrimination: e.g., "People have verbally abused } \\
\text { me or physically assaulted me" [38]. }\end{array}$ \\
\hline $\begin{array}{l}\text { Self-stigma } \\
\text { (anticipated) }\end{array}$ & $\begin{array}{l}\text { Expectation of experiencing prejudice and } \\
\text { discrimination due to a specific characteristic. In } \\
\text { surveys, (potentially) affected persons are asked. }\end{array}$ & $\begin{array}{l}\text { Fear, perceived consequences: "People would avoid } \\
\text { me because of my job" [37]. }\end{array}$ \\
\hline
\end{tabular}

\subsection{Information Sources and Search}

We searched the electronic databases Medline (via Pubmed), Embase (via Ovid) and PsycInfo (via EBSCOhost) until 23 October 2020. Moreover, we also searched the search engine of the ZB MED Information Centre for Life Science on 11 December 2020 and included results of a systematic search in the database CINAHL from a cooperating project on stigmatization in physicians and nurses (search date: 1 December 2020). The search strings are shown in the Supplementary Material, Table S1. In addition, the grey literature found while searching reference lists were included in this review (checked December 2020). We also used the "citation tracking function" by Google Scholar of the included studies to identify additional studies (January-February 2021).

\subsection{Study Selection and Data Collection}

Search results were imported into an Endnote reference management system database. Study selection was carried out in two steps. First, the screening of titles and abstracts was piloted with a random sample of 500 publications. Four raters (J.L., T.H., A.F. and M.S.) screened the records for eligibility criteria. Interrater reliability was moderate (Fleiss 
Kappa $=0.42)$ according to Landis and Koch [42] for the piloting phase. Thereafter, raters were assigned to $2 \times 2$ rater groups (J.L./A.F. and T.H./M.S.), and the remaining publications were divided equally between the two groups. The titles and abstracts were screened independently by two raters for inclusion and exclusion criteria. Disagreements regarding the inclusion were discussed and studies were included in the full-text screening to err on the side of caution if discussion was not resolved. Interrater reliability was moderate for both rater teams (Cohen's kappa $=0.49$, and 0.51). Second, the screening of all included full texts was carried out by the rater teams. Disagreements were solved by discussion in the research group. If a study was not included based on the full text screening, the reasons for exclusion were reported. The screening of full texts was piloted. For this, 20 randomly chosen publications were distributed to all raters, and interrater reliability was very good (Fleiss Kappa $=0.69$ ). Full texts were divided equally between the two groups and checked for eligibility. Interrater variability was very good for both rater teams (Cohen's kappa $=0.79$, and 0.81).

For the included studies, we extracted the following information from the publications in tables: title, author(s), publication year, study design, characteristics of the study population with job description, time of assessments, exposure, outcome measures and results. Further details regarding the study, such as the adjustment for confounders, ethical clearance, conflicts of interest, and funding sources were also extracted in the comments section of our extraction form. Data extraction was piloted. Data extraction was carried out by one reviewer of the team and checked by the other reviewer of the team (J.L./A.F. and T.H./M.S.).

\subsection{Rating of Methodological Study Quality (Risk of Bias)}

The risk of bias was assessed only for studies reporting stigmatization prevalence (including information on frequency/occurrence of stigmatization) or the prevalence of health consequences. The methodological study quality was determined according to Ijaz et al. [43] and Kuijer et al. [44] with modifications. This method has been used previously in other studies [45].

For the evaluation of the study quality, the following domains were used: (1) recruitment procedure and follow up (in cohort studies), (2) exposure definition and measurement, (3) outcome source and validation, (4) confounding and effect modification, (5) analysis method (methods to reduce research specific bias), (6) chronology, (7) blinding of assessors, (8) funding, and (9) conflict of interest. The quality assessment includes six major domains (1-6) and three minor domains (7-9). Per definition, an overall low risk of bias for the study was assumed if all major domains were rated low. Disagreements were solved by discussion. The methodological quality was independently assessed by at least two reviewers (J.L., A.F., T.H., or M.S.) for each study. Disagreements in quality ratings between the two raters were solved by discussion.

\subsection{Synthesis of Results and Meta-Analysis}

The study results were summarized descriptively and in meta-analyses. For estimating the prevalence of stigmatization, we only considered studies with an adequate sampling design. Thus, we excluded studies characterized by convenience sampling from results synthesis for the reason that assumptions of those studies are not generalizable.

Meta-analyses were performed to estimate the pooled risk of stigmatization on depression and anxiety. The meta-analysis was carried out if at least two primary studies which were comparable in terms of exposure and outcome were present. Due to the heterogeneity of the studies, random effects models were used as the analysis method. Since adjusted and unadjusted risk estimates were close to each other, we decided to include adjusted and unadjusted values in the meta-analysis. The software Stata Version 14.0 (StataCorp, College Station, TX, USA) was used. 


\section{Results}

\subsection{Study Selection}

We identified a total of 7452 records from the electronic database and preprint servers of which 167 full texts were assessed for eligibility. Furthermore, 33 additional records were identified from the grey literature search. We included a total of 46 articles in our systematic review. A total of 154 full-text articles were excluded from further consideration. The most common reason for exclusion was that the articles were editorials, comments, opinions, letters, or narrative reviews $(n=68)$. Furthermore, 34 articles were excluded because the exposure was related to other emerging respiratory virus diseases (such as MERS, SARS) and did not include COVID-19. In 30 cases, the outcome did not relate to stigmatization, and in another 10 cases, the study did not include the working population. Further articles were excluded for not being related to the topic $(n=5)$, exposure was not work $(n=2)$, poster presentation $(n=1)$ and being a duplicate $(n=1)$. In addition, despite extensive efforts by our librarian, we were unable to locate the full texts of three records. The literature search and all the reasons for the exclusion of full-text articles are summarized in the PRISMA flow diagram (Figure 1). Moreover, references of all included and excluded studies with the reason for exclusion are listed in the Supplementary Material Table S2.

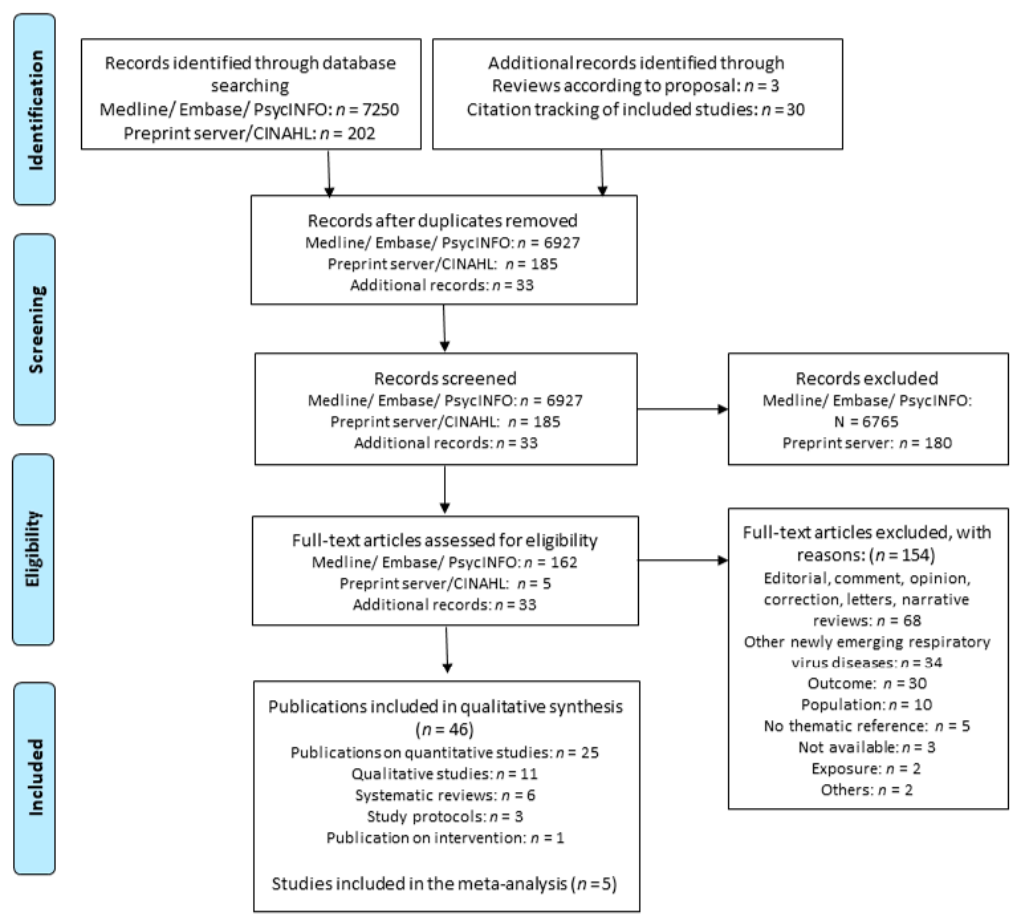

Figure 1. PRISMA flow diagram. Adapted from: Moher D, Liberati A, Tetzlaff J, Altman DG, the PRISMA Group (2009). Preferred reporting items for systematic reviews and meta-analyses: the PRISMA statement. PLoS Med 6(6): e1000097, doi:https://doi.org/10.1371/journal.pmed1000097.

\subsection{Study Characteristics}

A total of 46 articles examining associations of work-related COVID-19 exposure with the risk of stigmatization were included: 23 articles from 22 cross-sectional studies, 2 longitudinal studies and 11 interview studies. Further, we included six systematic reviews, three study protocols and one intervention study in our qualitative synthesis.

\subsubsection{Systematic Reviews}

We included six systematic reviews with original studies on work-related stigmatization due to COVID-19 exposure in our literature search [28,30,33,46-48]. Four of the reviews $[28,30,33,46]$ only included one original study in their review, which we all iden- 
tified by our systematic search (original studies: Blake et al. [49], Chatterjee et al. [50], Juan et al. [51], and Mohindra et al. [38]). Joo et al. [47] included the qualitative studies by Kackin et al. [52] and Kalateh-Sadati et al. [53] in their systematic review. Three studies on work-related COVID-19 stigmatization were included by Rahman et al. [48], of which two were excluded by us because they did not meet our inclusion criteria $[54,55]$. The qualitative study by Fawaz et al. [56] was identified by our search. Information on the original studies is provided in the following tables as well as in the Supplementary Table S3.

\subsubsection{Quantitative Studies}

We included 22 cross-sectional studies (23 articles) [35-38,40,41,50,51,57-71] and two longitudinal studies $[39,72]$ in our systematic review. Most studies were conducted in India $(n=5)[38,50,67-69]$ and China $(n=4)[41,51,66,72]$, followed by Vietnam $(n=2)[36,40]$ and Libya $(n=2)$ [58,59]. One study each was conducted in Canada [35], Colombia [63], Egypt [70], Italy [64], Iran [71], Nepal [61,62], Pakistan [37], Singapore [39], the UK [60], and the USA [65]. One study was conducted around the world [57]. The association of COVID-19 with stigmatization was predominately studied in HCWs, medical jobs (such as lab technicians, medical officers), and other jobs related to hospitals (attendants/cleaners, managers/clerks) (19 studies). One study [36] considered HCWs, professional educators and white collar workers, and another study [72] differentiated between governmental/public institutions and private enterprises. Tan et al. [66] investigated returning to work in workers/technical staff and executives, sales and marketing management, and others. Furthermore, Taylor and colleagues [35] investigated the attitudes from the general population towards HCWs, and only included non-HCWs. Another study [57] did not report any information on the work of participants. Besides the study by Said et al. which included only women [70], all studies included females and males. All studies were conducted during the COVID-19 pandemic, of which 13 studies did not give more detailed information concerning the epidemic/pandemic phase. Four studies were carried out during lockdown [36,38,61,62,64]. One study each was performed during the "rapid increase in COVID-19 cases and death", in the "post-peak phase" [60], at the "highest point" [51], during the "initial phase of containment" [63], or "two weeks after government suspended all public transports" [41]. In addition, one study was carried out after returning to work from lockdown and quarantine during COVID-19 peak [66]. Another study was conducted after the quarantine of employees working in a hospital that was locked down due to a COVID-19 outbreak [40]. Stigmatization was assessed with a wide range of different validated and non-validated instruments. Ten studies measured stigmatization with a single item [41,50,51,57-62,66,72]. Two studies each used 2-item [64,70], 4-item [36,37], 12-item [39,40], and 13-item questionnaires [67,68]. One study each used 8-item [35], 19-item [38] and 22-item questionnaires [71]. Yadav and colleagues [69] used an adapted stigma assessment and reduction of impact (SARI) stigma scale. Monterrossa-Castro et al. [63] and Sharma et al. [65] did not provide detailed information on the instrument used.

The majority of studies investigated perceived self-stigma (17 of 24 studies) which was commonly assessed via feelings of discrimination $(n=13)[40,51,61-64,66-72]$ and social exclusion $(n=5)[36,41,50,67,68]$. In addition, four studies investigated perceived aggressive/behaviour/bullying [38,57,61,62,69], and one study used stereotypes [38]. Further, anticipated self-stigma was determined in seven studies $[36,37,39,40,64,67,68]$. Internalized self-stigma and associative stigma (via social exclusion and aggression/bullying) was investigated in four studies each (associative stigma: $[36,57,69,73]$, internalized selfstigma: $[38-40,70])$. One study investigated public stigma towards HCWs in the general population, measuring stereotypes, discrimination and social exclusion [35].

The majority of studies comprised of convenience samples according to the sampling technique, or because the number of invited persons was not reported $(n=17)$. Only seven studies used an adequate sampling design $[39,41,51,58,66,69,71]$. All studies were considered of low methodological quality (i.e., high risk of bias). A short description of all studies is given in Table 4. The full extraction table is shown in the Supplementary File Table S4. 
Table 4. Summary of included quantitative studies.

\begin{tabular}{|c|c|c|c|c|}
\hline $\begin{array}{l}\text { First Author } \\
\text { Risk of Bias }\end{array}$ & $\begin{array}{l}\text { Region Study Design } \\
\text { Comments }\end{array}$ & Population & $\begin{array}{c}\text { Stigmatization Form } \\
\text { Assessment }\end{array}$ & Time of COVID-19 Pandemic \\
\hline $\begin{array}{l}\text { Chatterjee et al. [50] } \\
\text { High risk }\end{array}$ & $\begin{array}{l}\text { India } \\
\text { CS } \\
\text { Convenience sample }\end{array}$ & Physicians & $\begin{array}{l}\text { Self-stigma (perceived) } \\
\text { 1-item }\end{array}$ & Not specified \\
\hline $\begin{array}{l}\text { Chaudhary et al. [37] } \\
\text { High risk }\end{array}$ & $\begin{array}{l}\text { Pakistan } \\
\text { CS } \\
\text { \# invited n. r. }\end{array}$ & $\begin{array}{l}\text { Clinical oral HCWs } \\
\text { Non-clinical oral HCWs from } 10 \text { different } \\
\text { dental hospitals }\end{array}$ & $\begin{array}{l}\text { Self-stigma (anticipated) } \\
\text { Associative stigma } \\
\text { 4-items }\end{array}$ & Not specified \\
\hline Chen et al. [72] High risk & $\begin{array}{l}\text { China } \\
\text { Longitudinal } \\
\text { \# invited n. r. }\end{array}$ & $\begin{array}{l}\text { Government/public } \\
\text { institution/institutions/state-owned, enterprises, } \\
\text { private enterprise staff or individual business }\end{array}$ & $\begin{array}{l}\text { Self-stigma (perceived) } \\
\text { 1-item }\end{array}$ & $\begin{array}{l}\text { Baseline: rapid increase in COVID-19 cases } \\
\text { and related deaths; follow-up: authorities } \\
\text { relaxed lowdown }\end{array}$ \\
\hline $\begin{array}{l}\text { Chew et al. [39] } \\
\text { High risk }\end{array}$ & $\begin{array}{l}\text { Singapore } \\
\text { Longitudinal } \\
\text { Response: } 49.2 \%\end{array}$ & $\begin{array}{l}\text { Medical residents in training (medical } \\
\text { and surgical) }\end{array}$ & $\begin{array}{l}\text { Self-stigma (anticipated) } \\
\text { Self-stigma (internalized) } \\
12 \text { items (Healthcare Workers Stigma scale, HWSS) }\end{array}$ & Not specified \\
\hline $\begin{array}{l}\text { Dang et al. [36] } \\
\text { High risk }\end{array}$ & $\begin{array}{l}\text { Vietnam } \\
\text { CS } \\
\text { Convenience sample }\end{array}$ & $\begin{array}{l}\text { HCWs, professional educators, white collar } \\
\text { workers, students, others }\end{array}$ & $\begin{array}{l}\text { Self-stigma (perceived) } \\
\text { Self-stigma (anticipated) } \\
\text { Associative stigma } \\
\text { 4-items }\end{array}$ & $\begin{array}{l}\text { Data collection one week after social } \\
\text { distancing and lockdown was ordered } \\
\text { by government }\end{array}$ \\
\hline $\begin{array}{l}\text { Do Duy et al. [40] } \\
\text { High risk }\end{array}$ & $\begin{array}{l}\text { Vietnam } \\
\text { CS } \\
\text { \# invited n. r. }\end{array}$ & Clinicians, nurses, others & $\begin{array}{l}\text { Self-stigma (perceived) } \\
\text { Self-stigma (anticipated) } \\
\text { Self-stigma (internalized) } \\
12 \text { items (adaption of Berger's HIV Stigma Scale) }\end{array}$ & $\begin{array}{l}\text { Lockdown of workplace because of } \\
\text { COVID-19 outbreak-> all employees } \\
\text { required to quarantine for } 23 \text { days. Data } \\
\text { collection after quarantine }\end{array}$ \\
\hline $\begin{array}{l}\text { Dye et al. [57] } \\
\text { High risk }\end{array}$ & $\begin{array}{l}\text { Worldwide } \\
\text { CS } \\
\text { Convenience sample }\end{array}$ & n.r. & $\begin{array}{l}\text { Self-stigma (anticipated) } \\
\text { Associative stigma } \\
1 \text { item }\end{array}$ & Not specified \\
\hline $\begin{array}{l}\text { Elhadi et al. [59] } \\
\text { High risk }\end{array}$ & $\begin{array}{l}\text { Libya } \\
\text { CS } \\
\text { Convenience sample } \\
\end{array}$ & $\begin{array}{l}\text { HCWs (doctors and nurses) from } 15 \text { hospitals } \\
\text { working during the outbreak period }\end{array}$ & $\begin{array}{l}\text { Self-stigma (perceived) } \\
1 \text { item }\end{array}$ & $\begin{array}{l}\text { Not specified } \\
\text { (but during civil war) }\end{array}$ \\
\hline $\begin{array}{l}\text { Elhadi et al. [58] } \\
\text { High risk }\end{array}$ & $\begin{array}{l}\text { Libya } \\
\text { CS } \\
\text { Response: } 88.7 \% \\
\end{array}$ & $\begin{array}{l}\text { HCWs working in either surgery, internal } \\
\text { medicine, intensive care, or } \\
\text { emergency departments }\end{array}$ & $\begin{array}{l}\text { Self-stigma (perceived) } \\
1 \text { item }\end{array}$ & $\begin{array}{l}\text { Not specified } \\
\text { (but during civil war) }\end{array}$ \\
\hline $\begin{array}{l}\text { Greene et al. [60] } \\
\text { High risk }\end{array}$ & $\begin{array}{l}\text { UK } \\
\text { CS } \\
\text { Convenience sample }\end{array}$ & $\begin{array}{l}\text { Frontline health and social care workers working } \\
\text { in a variety of healthcare roles in UK hospitals, } \\
\text { nursing or care homes, and community settings }\end{array}$ & $\begin{array}{l}\text { Self-stigma (perceived) } \\
1 \text { item }\end{array}$ & $\begin{array}{l}\text { During COVID-19 pandemic (post-peak } \\
\text { phase of the initial COVID-19 wave in } \\
\text { the UK) }\end{array}$ \\
\hline $\begin{array}{l}\text { Juan et al. [51] } \\
\text { High risk }\end{array}$ & $\begin{array}{l}\text { China } \\
\text { CS } \\
\text { Response: } 91.2 \%\end{array}$ & $\begin{array}{l}\text { hospital staff from five national COVID-19 } \\
\text { designated hospitals (working in isolation ward, } \\
\text { general ward) }\end{array}$ & $\begin{array}{l}\text { Self-stigma (perceived) } \\
1 \text { item }\end{array}$ & $\begin{array}{l}\text { Study period corresponds with the highest } \\
\text { point of the COVID-19 epidemic in } \\
\text { China }\end{array}$ \\
\hline
\end{tabular}


Table 4. Cont.

\begin{tabular}{|c|c|c|c|c|}
\hline $\begin{array}{l}\text { First Author } \\
\text { Risk of Bias }\end{array}$ & $\begin{array}{l}\text { Region Study Design } \\
\text { Comments }\end{array}$ & Population & $\begin{array}{l}\text { Stigmatization Form } \\
\text { Assessment }\end{array}$ & Time of COVID-19 Pandemic \\
\hline $\begin{array}{l}\text { Khanal et al. [61,62] } \\
\text { High risk }\end{array}$ & $\begin{array}{l}\text { Nepal } \\
\text { CS } \\
\text { \# invited n. r. }\end{array}$ & $\begin{array}{l}\text { Nurses, doctors, paramedics, laboratory staff, } \\
\text { pharmacists, public health professional currently } \\
\text { working in COVID-19 management }\end{array}$ & $\begin{array}{l}\text { Self-stigma (perceived) } \\
1 \text { item }\end{array}$ & During lockdown \\
\hline $\begin{array}{l}\text { Mohindra et al. [38] } \\
\text { High risk }\end{array}$ & $\begin{array}{l}\text { India } \\
\text { CS } \\
\text { Convenience sample }\end{array}$ & $\begin{array}{l}\text { Doctors, nurses, hospital attendants, sanitation } \\
\text { attendants, others working at the hospital }\end{array}$ & $\begin{array}{l}\text { Self-stigma (perceived) } \\
\text { Self-stigma (internalized) } \\
19 \text { items (adapted from Ebola epidemic } \\
\text { questionnaire [74]) }\end{array}$ & During lockdown \\
\hline $\begin{array}{l}\text { Ramaci et al. [64] } \\
\text { High risk }\end{array}$ & $\begin{array}{l}\text { Italy } \\
\text { CS } \\
\text { Convenience sample }\end{array}$ & Nurses and doctors & $\begin{array}{l}\text { Self-stigma (perceived) } \\
\text { Self-stigma (anticipated) } \\
\text { Questionnaire adapted from HIV / AIDS/drug users } \\
\text { questionnaire [75] }\end{array}$ & During national lockdown \\
\hline $\begin{array}{l}\text { Said et al. [70] } \\
\text { High risk }\end{array}$ & $\begin{array}{l}\text { Egypt } \\
\text { CS (controlled) } \\
\text { Convenience sample }\end{array}$ & $\begin{array}{l}\text { Nurses from triage hospital and from a hospital } \\
\text { with no triage or isolation }\end{array}$ & $\begin{array}{l}\text { Self-stigma (perceived) } \\
\text { Self-stigma (internalized) } \\
2 \text { items (from US National Centre for Posttraumatic } \\
\text { Stress Disorder } 2020 \text { and "MERS-CoV } \\
\text { staff questionnaire") }\end{array}$ & Not specified \\
\hline $\begin{array}{l}\text { Sharma et al. [65] } \\
\text { High risk }\end{array}$ & $\begin{array}{l}\text { USA } \\
\text { CS } \\
\text { Convenience sample }\end{array}$ & $\begin{array}{l}\text { HCWs caring for COVID-19 patients (intensive } \\
\text { care unit): physicians, nurses, respiratory } \\
\text { therapists, advanced practice providers }\end{array}$ & $\begin{array}{l}\text { Self-stigma (perceived) } \\
\text { Not reported }\end{array}$ & Not specified \\
\hline $\begin{array}{l}\text { Tan et al. [66] } \\
\text { High risk }\end{array}$ & $\begin{array}{l}\text { China } \\
\text { CS } \\
\text { Response: } 50.9 \%\end{array}$ & $\begin{array}{l}\text { Members of the workforce who returned to work: } \\
\text { workers, and technical staff, executives, sales and } \\
\text { marketing, management and others }\end{array}$ & $\begin{array}{l}\text { Self-stigma (perceived) } \\
1 \text { item }\end{array}$ & $\begin{array}{l}\text { Returning to work after lockdown and } \\
\text { quarantine in Chongqing, during the peak of } \\
\text { the COVID-19 epidemic when strict infection } \\
\text { control was in place }\end{array}$ \\
\hline $\begin{array}{l}\text { Taylor et al. [35] } \\
\text { High risk }\end{array}$ & $\begin{array}{l}\text { Canada, USA } \\
\text { CS } \\
\# \text { invited n. r. }\end{array}$ & Non-HCWs & $\begin{array}{l}\text { Public stigma } \\
8 \text { items }\end{array}$ & Not specified \\
\hline $\begin{array}{l}\text { Uvais et al. }[67,68] \\
\text { High risk }\end{array}$ & $\begin{array}{l}\text { India } \\
\text { CS } \\
\text { Convenience sample }\end{array}$ & Physicians working in hospitals & $\begin{array}{l}\text { Self-stigma (perceived) } \\
\text { Self-stigma (anticipated) } \\
13 \text { items (Perceived Stigma Scale) }\end{array}$ & Not specified \\
\hline $\begin{array}{l}\text { Yadav et al. [69] } \\
\text { High risk }\end{array}$ & $\begin{array}{l}\text { India } \\
\text { CS } \\
\text { Response: } 36.6 \%\end{array}$ & HCWs & $\begin{array}{l}\text { Self-stigma (perceived) } \\
\text { Associative stigma } \\
\text { Adapted Stigma assessment and reduction of impact } \\
\text { (SARI) Stigma scale }\end{array}$ & Not specified \\
\hline
\end{tabular}


Table 4. Cont.

\begin{tabular}{|c|c|c|c|c|}
\hline $\begin{array}{l}\text { First Author } \\
\text { Risk of Bias }\end{array}$ & $\begin{array}{l}\text { Region Study Design } \\
\text { Comments }\end{array}$ & Population & $\begin{array}{c}\text { Stigmatization Form } \\
\text { Assessment }\end{array}$ & Time of COVID-19 Pandemic \\
\hline $\begin{array}{l}\text { Zandifar et al. [71] } \\
\text { High risk }\end{array}$ & $\begin{array}{l}\text { Iran } \\
\text { CS } \\
\text { Response: } 92 \%\end{array}$ & $\begin{array}{l}\text { HCWs engaged in the field of diagnostic and } \\
\text { treatment of COVID-19 patients working in } 9 \\
\text { general hospitals (physicians, } \\
\text { nurses, technicians) }\end{array}$ & $\begin{array}{l}\text { Self-stigma (perceived) } \\
\text { 22-items (adopted from the HIV Stigma Scale) }\end{array}$ & Not specified \\
\hline $\begin{array}{l}\text { Zhu et al. [41] } \\
\text { High risk }\end{array}$ & $\begin{array}{l}\text { China } \\
\text { CS } \\
\text { Response: } 77.1 \%\end{array}$ & $\begin{array}{l}\text { HCWs from hospital directly providing services } \\
\text { to confirmed or suspected COVID-19 patients } \\
\text { (physicians, nurses, technicians) }\end{array}$ & $\begin{array}{l}\text { Self-stigma (perceived) } \\
1 \text { item }\end{array}$ & $\begin{array}{l}\text { COVID-19 outbreak ( } 2 \text { weeks after the } \\
\text { authority in Wuhan suspended all } \\
\text { public transport) }\end{array}$ \\
\hline
\end{tabular}

\# invited n. r. = number of invited participants was not reported, CS = cross-sectional study, longitudinal = longitudinal study design. 


\subsubsection{Qualitative Studies}

We included 11 qualitative studies and one cross-sectional study with a qualitative part in this systematic review. Studies were conducted in Iran [53,76] and Pakistan $[77,78]$ ( $n=2$ each) as well as in Canada [79], Germany [80], Lebanon [56], Nepal [81], South Korea [82], and Turkey [52] ( $n=1$ each). The USA, Kenya, Ireland and Canada [83] were presented by one study each. Most studies included working males and females $(n=7)$. Two studies only interviewed female nurses $[79,82]$. Another two studies did not provide any information concerning the gender of participants $[53,56]$. Nearly all studies were performed in HCWs. Six studies included nurses only, while one study interviewed physicians and nurses [56]. Another study also included the hospital management involved in COVID-19 management in addition to physicians and nurses [78]. Crowe et al. [79] interviewed nurses and employees from high acuity units in an academic teaching hospital. Zolnikov et al. [83] interviewed first responders including HCWs, firefighters, paramedics, police officers, and technicians. Bhatt and colleagues [81] included individuals working at the forefront in the community (HCWs, police officers, school teachers). One study did not give a job description [77]. In addition, we included results of the cross-sectional study by Dye et al. [57] which consists of a qualitative part. For a general description of this study, see Table 5.

Perceived self-stigma was assessed in 11 of 12 studies. In addition, five studies determined associative stigma [52,57,80-82]. One study each investigated anticipated self-stigma [78] and internalized self-stigma (use of negative words "feeling dirty", "feeling "contaminated") [83].

Full data extraction of studies is provided in Table S5.

\subsubsection{Intervention}

We included one study that rapidly developed a free digital learning package for promoting mental health in UK healthcare employees in response to the COVID-19 pandemic. [49]. For a description of the results, see Section 3.4.

\subsubsection{Study Protocols}

Three study protocols were included in this systematic review. One study protocol outlines the procedure of a scoping review for a systematic literature search on people with disabilities or other vulnerabilities during the COVID-19 pandemic [84]. The review has not been published so far (checked: 22 April 2021).

The second study protocol describes the procedure for a repeated cross-sectional study on the mental health of HCWs and the general public during COVID-19 in Thailand (study name: HOME-COVID-19) [85]. The study aims to assess public stigma towards COVID-19 infections. The results of a single wave have been published in two studies [86,87], but work-related stigmatization due to COVID-19 exposure has not been investigated yet.

The third publication presents a protocol for guideline for "Psychological First Aid (PFA)" in HCWs in Malaysia [88]. 
Table 5. Summary of included qualitative studies.

\begin{tabular}{|c|c|c|c|c|}
\hline Study & Region & Population & Stigmatization Form Assessment & Time of COVID-19 Pandemic \\
\hline Bhatt et al. [81] & Nepal & $\begin{array}{l}\text { Teachers, students, security personnel, head of household, } \\
\text { leaders, health workers, homemaker, others }\end{array}$ & $\begin{array}{l}\text { Self-stigma (perceived) } \\
\text { Associative stigma } \\
\text { Interviews and focus group discussions }\end{array}$ & Not specified \\
\hline Dye et al. [57] & Worldwide & Not reported & $\begin{array}{l}\text { Self-stigma (perceived) } \\
\text { Associative stigma } \\
\text { Open-ended question }\end{array}$ & Not specified \\
\hline Fawaz et al. [56] & Lebanon & Nurses and physicians working at various COVID-19 units & $\begin{array}{l}\text { Self-stigma (perceived) } \\
\text { Semi-structured interviews }\end{array}$ & $\begin{array}{l}\text { Being quarantined following occupational } \\
\text { COVID-19 exposure }\end{array}$ \\
\hline Feroz et al. [78] & Pakistan & $\begin{array}{l}\text { Key informants KIIs (senior management and hospital } \\
\text { leadership, directly or indirectly involved with the } \\
\text { management of COVID-19 patients) }\end{array}$ & $\begin{array}{l}\text { Self-stigma (anticipated) } \\
\text { Semi-structured interviews and a purposive } \\
\text { sampling approach }\end{array}$ & Not specified \\
\hline Hien et al. [80] & Germany & Nurses in clinics and retirement homes & $\begin{array}{l}\text { Self-stigma (perceived) } \\
\text { Associative stigma } \\
\text { Interviews }\end{array}$ & Not specified \\
\hline Kackin et al. [52] & Turkey & Nurses caring for COVID-19 patients & $\begin{array}{l}\text { Self-stigma (perceived) } \\
\text { Associative stigma } \\
\text { Semi-structured interviews }\end{array}$ & Not specified \\
\hline Kalateh-Sadati et al. [53] & Iran & $\begin{array}{l}\text { Nurses working in hospitals specified for } \\
\text { COVID-19 treatment }\end{array}$ & $\begin{array}{l}\text { Self-stigma (perceived) } \\
\text { Interviews }\end{array}$ & Not specified \\
\hline Lee et al. [82] & South Korea & $\begin{array}{l}\text { COVID-19-designated hospital nurses providing direct care } \\
\text { for patients }\end{array}$ & $\begin{array}{l}\text { Self-stigma (perceived) } \\
\text { Associative stigma } \\
\text { In-depth interviews }\end{array}$ & Not specified \\
\hline Reazee et al. [76] & Iran & Nurses working fulltime in COVID-19 wards & $\begin{array}{l}\text { Self-stigma (perceived) } \\
\text { Associative stigma } \\
\text { Interviews }\end{array}$ & Not specified \\
\hline Rizvi Jafree et al. [77] & Pakistan & Not reported & $\begin{array}{l}\text { Self-stigma (perceived) } \\
\text { Semi-structured interviews }\end{array}$ & $\begin{array}{l}\text { COVID-19-affected families admitted at three } \\
\text { government-allocated hospitals }\end{array}$ \\
\hline Zolnikov et al. [83] & $\begin{array}{l}\text { Canada, Ireland, } \\
\text { Kenya, USA }\end{array}$ & $\begin{array}{l}\text { First responders/HCWs: nurses, physicians, firefighters, } \\
\text { paramedics, police officers, nurse technicians, behavioural } \\
\text { therapists, orthodontists, dialysis technicians, technicians in } \\
\text { medical surgery, data specialists, emergency } \\
\text { medical technicians }\end{array}$ & $\begin{array}{l}\text { Self-stigma (internalized) } \\
\text { Semi-structured interviews }\end{array}$ & Not specified \\
\hline
\end{tabular}




\subsection{Prevalance of Stigmatization with Regard to Occupational Group}

\subsubsection{Quantitative Studies}

For evaluating the prevalence of stigmatization, only studies with an adequate sampling design were considered $(n=7)$. Studies characterized by an adequate sampling design were mostly from China $(n=3)[41,51,66]$; one study each was from India [69], Iran [71], Libya [58], and Singapore [39]. The responses ranged from $37-92 \%$. All but the study by Tan et al. [66] investigated stigmatization in HCWs, or hospital-related jobs.

Six studies measured perceived self-stigma: Zhu et al. [41] found that $19.5 \%$ of the participants felt avoided by family and friends (social exclusion). Yadav et al. [69] described a similar prevalence of $19.3 \%$ for perceived self-stigma, of which the majority (70\%) experienced rude behaviour by community, followed by racial/obscene/derogatory remarks (32\%) and harassment by their landlord and neighbours (32\%). In addition, $13 \%$ felt harassed by security personnel. Moreover, more resident doctors and nurses perceived stigma than faculty/medical officers. Elhadi and colleagues [58] found that $31.8 \%$ of HCWs working in Libyan hospitals felt stigmatized, and this was higher in female than in male HCWs (36.1\% versus $28.2 \%$ ). The study by Tan et al. [66] investigated perceived self-stigma in workers and technical staff, executives, sales and marketing management returning to work after lockdown and quarantine. Perceived discrimination (moderate to very serious) was lower in management and executive staff than in workers/technical staff ( $0.8 \%$ versus $3.5 \%$ ). Zandifar et al. [71] used a modified HIV Stigma Scale and presented their results on perceived self-stigma (discrimination) using the median without the classification of values. Stigmatization was highest for physicians (median $=29$ ), followed by nurses (26), and technicians (22). Juan et al. [51] investigated psychological distress in hospital staff in association with perceived discrimination during the highest point of the epidemic in China but did not give information on the prevalence. A longitudinal study by Chew et al. [39] measured anticipated and internalized self-stigma using a 12-item healthcare worker stigma scale. The results were presented as means and the authors showed that anticipated and internalized self-stigma was higher at baseline during the COVID-19 pandemic compared to the follow-up after 3 months. Stigma (HWSS total score) was not significantly associated with being exposed to patients with respiratory disease $(B=0.451, p=0.656)$. Associative stigma was only studied by Yadav et al. [69] and about $11.8 \%$ of family members were affected by this. Detailed results of all studies are shown in the extraction tables in the Supplementary Table S4.

\subsubsection{Qualitative Studies}

The results indicate that HCWs and their families are especially prone to stigmatization in daily life. Participants and their relatives reported being harassed, attacked and bullied by neighbours and community who perceived them and their family as "coronainfected". Moreover, HCWs were not allowed to enter a supermarket [57], or to use public transport/taxi $[53,82]$. Furthermore, they were asked to leave the apartment $[57,81]$ and were also denied proper food and lodging in hotels [81]. Comments in the news questioned why HCWs go out to eat or to the gym [82]. One female nurse reported that people were yelling at her for being too close and for being outside walking the dog [79]. Another female reported to be sneezed on at very close distance on purpose for enforcing physical distancing at work [57]. Zolnikov and colleagues [83] also showed other aggressive public reactions, including a person pulling down her mask and coughing at the first responders. In two studies, nurses reported that their husbands were not allowed at their work $[77,80]$. At work, a nurse became the target of criticism (flooding of social network posts) when she was diagnosed with COVID-19 [82]. Moreover, one physician reported being treated as suspicious by other physicians despite negative test results and the strict appliance of personal protective equipment measures [77]. Another participant reported that the work contract of his son who got COVID-19 on duty as a security officer in a bank was not resumed [77]. 
HCWs / first responders do not only face stigmatization from the community /workplace but also from friends and family $[52,53,56,76,79,83]$. Most frequently, HCWs reported being "treated like a virus", and were excluded by family and friends, leading to alienation. Reactions were more exaggerated when it was known that the person worked in the COVID-19 unit. Additionally, in one study, the participant was asked by a family member whether he was short in money caring for COVID-19-patients and was recommended to leave the job [76].

As a result, HCWs hide information about working with COVID-19 patients from family and community $[78,82]$. Due to the negative impact on social relationships, participants were feeling isolated and annoyed. Some participants also reported increased feelings of frustration and anger [56,82], thinking about quitting [82], or to not work in the COVID-19 ward any further [76]. A detailed extraction of studies is provided in the Supplementary File S5.

\subsection{Stigmatization from Work-Related COVID-19 Exposure and Health}

\subsubsection{Descriptive Summary}

Associations of work-related stigmatization from COVID-19 exposure with health was investigated in 14 studies [35,39-41,50,51,58-61,63-65,68,71,83]. All but one study [83] were quantitative studies. Studies assessed a variety of psychological disorders (Supplementary Table S6). Greene et al. [60], Juan et al. [51], Monterrossa-Castro et al. [63], and Zhu et al. [41] used the 7-item Generalized Anxiety Disorders Questionnaire (GAD-7) for assessing anxiety disorders. The authors showed that anxiety was significantly increased in association with being stigmatized. This was also true for depressive symptoms measured with the Patient Health Questionnaire 9 (PHQ-9, $n=3$ ) [41,51,60]. The Depression, Anxiety, and Stress Scale (DASS-21) was used in two studies [40,50]. The Hospital Anxiety and Depression Scale (HADS) was also used in two studies [59,61]. Studies found a significant association between psychological symptoms and being stigmatized. In addition, Chew et al. [39], Juan et al. [51], and Zhu et al. [41] studied the experience of traumatic events using the Impact of Event Scale-Revised (IES-R) and found increased traumatic stress in association with being stigmatized due to work-related COVID-19 exposure. Further, perceived self-stigma was associated with a higher risk of post-traumatic stress disorders (PTSD), measured with the PTSD subscale of the International Trauma Questionnaire (ITQ) [60], or the Posttraumatic Stress Disorder 8-item Questionnaire (PTSD-8) [71]. Elhadi and colleagues [58] used an abridged version of the Maslach Burnout Inventory (MBI), and showed that emotional exhaustion was significantly correlated with feeling stigmatized. Higher perceived stress using the Perceived Stress Scale (PSS-10) was shown by two further studies [39,68]. Taylor et al. [35] studied the COVID Stress Syndrome (CSS) with a selfdeveloped 5-item scale. The Quality of Life Scale (ProQOL) was used by Ramaci et al. [64]. Here, perceived and anticipated stigma was related to higher levels of compassion fatigue and burnout as well as lower compassion satisfaction. In addition, Juan and colleagues [51] investigated associations of stigmatization and rejection in the neighbourhood because of hospital workers with obsessive compulsive symptoms using the Yale-Brown ObsessiveCompulsive Scale (Y-BOCS) and the Patient Health Questionnaire 15 (PHQ-15) but found no significant relationship. Further, a significant association between stigmatization and insomnia (measured with the Insomnia Severity Index (ISI)) was found by Khanal et al. [61]. Sharma et al. [65] found increased emotional distress/burnout in association with stigma from community in HCWs but did not describe the instrument used.

In the interviews by Zolnikov et al. [83], participants reported negative feelings, stress, alcohol usage in association with stigmatization. Instruments and results on health effects reported in the original studies are shown in Table S6.

\subsubsection{Synthesis of Results}

Four studies reported risk estimates for depression (Figure 2A) and five studies for anxiety (Figure 2B) with regard to work-related stigmatization. The prevalence of 
depression varied: $13.5 \%$ [ 41,61$]$ over $29.6 \%$ [51] to $46.9 \%$ [60]. The prevalence of anxiety varied from $18.3 \%$ [61], $24.1 \%$ [41], 31.6\% [51], 39.3\% [63] to $47.3 \%$ [60].

\section{(A)}

Risk of depression

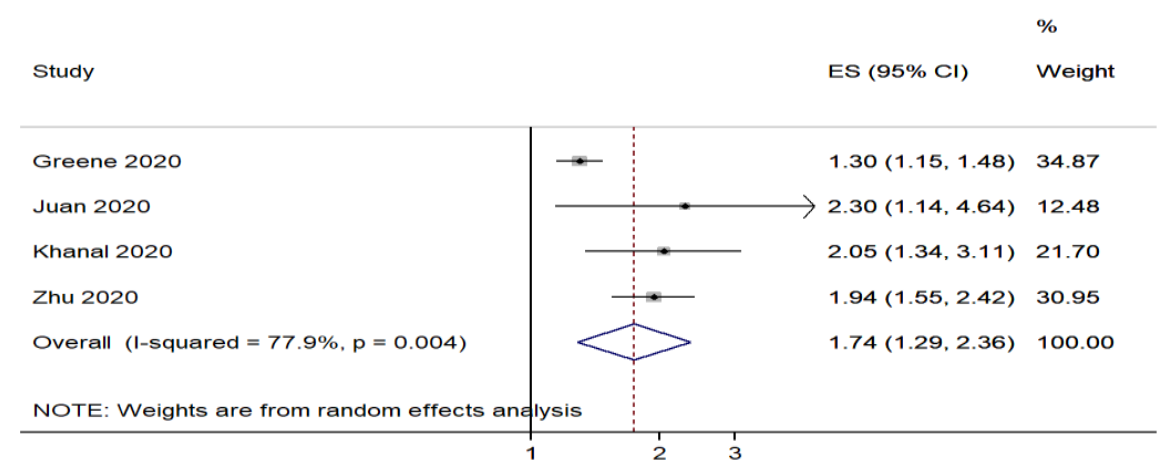

(B)

Risk of anxiety

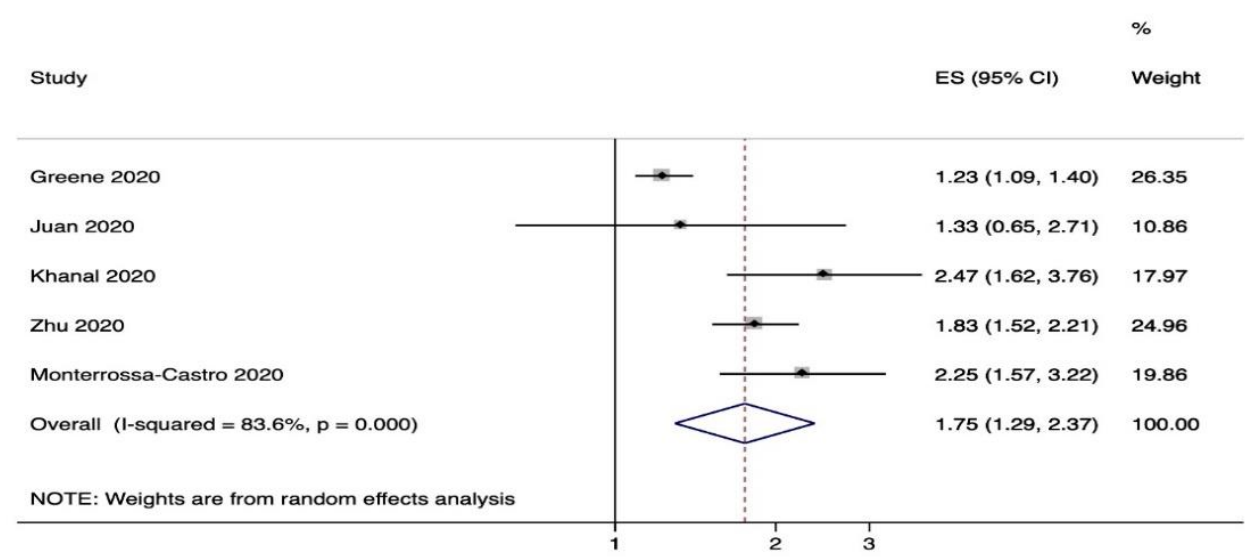

Figure 2. Risk of depression (A) and anxiety (B) in association with work-related stigmatization from COVID-19 exposure.

The results of the meta-analyses indicate a significant association between the workrelated stigmatization of HCWs and symptoms of depression and anxiety. In detail, the odds for depression were significantly increased by $74 \%(\mathrm{OR}=1.74 ; 95 \%$ CI 1.29 2.36 , Figure $2 \mathrm{~A})$ and anxiety by $75 \%(\mathrm{OR}=1.75 ; 95 \% \mathrm{CI} 1.29-2.37$, Figure $2 \mathrm{~B})$ for HCWs experiencing work-related stigmatization.

\subsection{Measures to Prevent Work-Related Stigmatization from COVID-19}

There was no quantitative study focussing on measures increasing or preventing workrelated stigmatization. In the qualitative study by Zolnikov and colleagues [83], participants were interviewed regarding measures which either increase or prevent work-related stigmatization. Good communication among colleagues was considered an important measure to prevent/deal with stigmatization at work. Further, keeping the connection between HCWs / first responders with those outside their professional roles was considered very important. Additionally, education and the dissemination of science-based information related to COVID-19 was deemed an important potential solution to fight stigmatization due to COVID-19 exposure. On the contrary, the distribution of misinformation (e.g., via social media) contributes to higher work-related stigmatization.

Furthermore, the study by Blake et al. [49] introduced a free online tool for sustaining mental health during the pandemic for UK HCWs. The development included a three-step process: 1. "public involvement activities", 2. "iterative peer review", and 3. "delivery" 
(measured as the number of users within 7 days after launch). The package was designed to offer actions team leaders can take for promoting mental health in staff and to provide guidance. The package includes the following topics: psychological impact, psychologically supportive teams, communication, social support, self-care, managing emotions, and further resources. In the section "communication", social stigma is addressed amongst other things. After the first 7 days of release, the package was accessed 17,633 times. A total of 55 participants completed the evaluation (49 HCWs and six students). The results indicate that the package has a high user satisfaction and that HCWs adopted the guidance in their daily life, e.g., taking further actions to emotionally support colleagues, consideration of psychological first aid training, or calling a telephone helpline. The package is provided at: https:/ / www.nottingham.ac.uk/toolkits / play_22794\#resume=1 (accessed on 3 June 2021).

Sulaiman and colleagues [88] developed a protocol for a "Psychological First Aid (PFA)" guideline for Malaysian HCWs affected by COVID-19 or suspected to be infected. The aid is based on the guidelines from the International Federation of Red Crescent Societies ("Look, Listen, Link") and employs a mobile application and phone calls. The aid is applied in a "Specific, Measurable, Attainable, Relevant, and Realistic Timeframe" (SMART) and contains five stages: (1) input-expert team set up and protocol development, (2) process-PFA-training and service promotion, (3) output-implementation, (4) outcomes-improvement of HCWs health, and (5) aim-evaluation of the PDA protocol. One objective is to encourage HCWs to get support from colleagues and employers, and to minimize stigma (core action: connection with social support). Information on the psychological effects resulting from work-related COVID-19 exposure and assistance for help are provided on the official website and via the promotion of social media, and posters.

\section{Discussion}

In summary, 46 articles examining stigmatization (mostly measured as perceived self-stigma) in the context of work-related COVID-19 exposure (mostly in HCWs) were included in this systematic review. Generally, all included studies indicate that stigmatization occurs as a result of work-related COVID-19 exposure. From a qualitative perspective, HCWs and their families suffer from stigmatization by the community and their work environment. Moreover, several reports indicate that HCWs are also discriminated against and socially excluded by family and friends. As a result, HCWs may not disclose information such as working with COVID-19 patients from their environment to reduce the impact of stigma [78,82]. This may lower feelings of belonging and social integration [89], as well as the usage of professional help [16]. However, selective disclosure may also limit stigmatization and ensure social support [90].

Most quantitative studies were characterized by convenience sampling (17 of 24). Since the results of these studies might be severely biased and do not allow for general conclusions, we did not include them in our result synthesis. Studies with an adequate sampling design $(n=7)$ were considered of low methodological quality, and only reported stigmatization prevalence percentage. Thus, results are hardly comparable and due to missing comparison groups, risk estimates cannot be determined. Descriptively, about 19\% of HCWs felt avoided by family and friends [41]. Perceived self-stigma from the community/neighbourhood was perceived by 19\% [69], respectively, by 32\% [58]. Associative stigma was perceived by $12 \%$ [69]. Moreover, in the study by Elhadi et al. [58], female HCWs felt more stigmatized than male HCWs (36.1\% versus $28.2 \%$ ). However, so far, there is no conclusive evidence for gender differences in the perception of stigma [91-93]. Furthermore, studies indicate that stigmatization was higher in doctors and nurses as compared to technicians / faculty officers [69,71]. Chew and colleagues [39] showed that stigma was not related to being exposed to patients with respiratory disease. Thus, work-related stigmatization from the community may be directed against an occupational group per se regardless of having actual contact to COVID-19 patients or not (as this is usually not known by the community). Occupational stigma has typically focused on "dirty work" [94]. 
Thus, working in a hospital per se may be valued as more "dirty" in response to the COVID19 pandemic than before. Shifts of occupational stigma during the pandemic have been especially observed in service workers. Whereas service work has been conceived as "dirty" before the pandemic, they have been put in the status of a "working hero" nowadays [95].

Associations of work-related stigmatization due to COVID-19 exposure with health were investigated in 14 studies, mainly by measuring symptoms of depression, anxiety, perceived stress, burnout, PTSD, insomnia, obsessive-compulsive behaviour, and somatization. The results for depression (measured with the PHQ-9) and generalized anxiety disorders (measured with the GAD-7) were summarized by meta-analysis. Work-related stigmatization from COVID-19 exposure significantly increases the risk for depression $(\mathrm{OR}=1.74 ; 95 \% \mathrm{CI} 1.29-2.36)$ and anxiety $(\mathrm{OR}=1.75 ; 95 \% \mathrm{CI} 1.29-2.37)$. The results are in line with recent systematic reviews on the psychological effects of providing healthcare during viral epidemic outbreaks $[25,26]$. Here, stigmatization has been shown to be a risk factor for acute and post-traumatic stress and psychological distress in HCWs caring for MERS-CoV2/SARS-patients. Additionally, a recently published cross-sectional study by Hennein et al. [96] on work-related stigmatization experiences in US HCWs shows similar results for major depression $(\mathrm{OR}=1.49,95 \% \mathrm{CI} 1.28-1.74)$ and generalized anxiety disorders $(\mathrm{OR}=1.39,95 \%$ CI 1.19-1.61), although disease prevalence was higher in our pooled analysis. Thus, in addition to the direct consequences of the COVID-19 pandemic, stigmatization further leads to an additional burden for workers.

We identified one qualitative study on conditions increasing/preventing stigmatization at work. The results indicate that communication, education and science-based information present useful tools to prevent work-related stigmatization. In addition, in the UK, a free online tool offers actions that team leaders can take for promoting mental health in staff and to provide guidance [49]. Since stigmatization is shown from diverse sources including the community, workplace and the social environment, anti-stigma strategies should be implemented at different levels including intra- and interpersonal, organizational/institutional, community and governmental/structural levels [97]. In addition, the International Labour Organization [98] developed a guideline to manage work-related psychological burden arising from the COVID-19 pandemic. This includes a section for how to deal with violence and harassment at work (which might be triggered by stigma). The actions are: (1) developing a workplace policy on violence and harassment, (2) implementing measures to protect workers from third party violence, (3) providing clear instruction how to defuse hostile situations, (4) establishing procedures for preventing the discrimination and harassment of workers in general and in particular workers infected with COVID-19, and (5) awareness of domestic violence. Employers should implement these actions for protecting the mental and physical health of the workforce. Furthermore, since research on anti-stigma strategies and their effectiveness is still very limited, we encourage future research to evaluate interventions for preventing work-related stigmatization.

The strengths of this systematic review are the systematic search in three databases with the forward and backward grey literature search of included studies focussing on COVID-19, and the presentation of data extraction in detail. Additionally, we have used a theory-based categorization of different stigma forms providing the basis for tailor-made work-related prevention programs. In addition, we have evaluated the included studies for their methodological quality for detecting a potential bias of the results. Study quality was a major problem, and we encourage future research to incorporate higher quality standards for sampling of the study population, the use of validated questionnaires, and the use of adequate comparison groups. The use of cohort studies as a study design is desirable. Moreover, since studies were predominately limited to HCWs, we further encourage future research on diverse occupations.

A limitation of this systematic review is the inclusion of publications from all over the world without taking cultural differences into account. Stigma is considered a cultural phenomenon, and global differences in stigmatization have been described [99]. Thus, we encourage future research to include cultural differences in the study of stigmatization 
due to work-related COVID-19 exposure and associated health consequences. In addition, since we searched electronic databases until October 2020, we were not able to investigate the influence of COVID-19 self-testing and the availability of vaccination on stigmatizing attitudes or on perceived stigma. Future studies on this topic are desirable.

\section{Conclusions}

As far as we are concerned, this is the first systematic review with a comprehensive overview of studies on work-related stigmatization in association with COVID-19 only. However, the scientific value of most studies is very limited, because the study population was recruited by convenience sampling, or because studies were characterized by low methodological quality. Additionally, studies used different techniques for assessing stigmatization, and a comparison group was missing. Thus, we encourage future research for adopting higher methodological standards for conducting and reporting of studies. This would provide a good basis for a review update limited to adequate studies in the future.

Regardless of the difficulties of reliably quantifying the extent of stigma due to methodological deficiencies in the studies, the stigmatization of occupations with contact to COVID19 or suspected patients is a relevant problem. We found clear evidence of the psychological consequences of COVID-19-related stigmatization for depression and anxiety disorders. For promoting workers' health, anti-stigma strategies and psychological support should be implemented in the workplace.

Supplementary Materials: The following are available online at https:/ /www.mdpi.com/article/ 10.3390/ijerph18126183/s1, Table S1: search strings, Table S2: included and excluded references, Table S3: extraction of included systematic reviews, Table S4: extraction of included quantitative studies, Table S5: extraction of included qualitative studies, Table S6: health effects of stigmatization.

Author Contributions: Conceptualization, A.S., A.F., J.L., O.v.d.K. and M.S.; methodology, G.F., C.A.; screening and extraction of data A.F., J.L., T.M.H., M.G. and M.S.; formal analysis, K.R.S.; writing—original draft preparation, M.S., J.L.; writing—review and editing, A.F., T.M.H., K.R.S., M.G., G.F., C.A., O.v.d.K., A.S., and M.S.; funding acquisition, A.S.; A.F., J.L., O.v.d.K., G.F., C.A., and M.S. All authors have read and agreed to the published version of the manuscript.

Funding: This research was funded by the Federal Institute for Occupational Safety and Health (Bundesanstalt für Arbeitsschutz und Arbeitsmedizin, BAuA), grant number F 2516.

Institutional Review Board Statement: Not applicable.

Informed Consent Statement: Not applicable.

Data Availability Statement: We did not collect own data for this systematic review, and our analyses are based on already published data. Data presented in this study are available in the supplementary material (i.e., extraction tables).

Acknowledgments: Our heartfelt thanks go to Soja Nazarov for help with acquiring hard to find literature. Special thanks to Uta Wegewitz and Marlen Melzer for their support of the study.

Conflicts of Interest: The authors declare no conflict of interest. The funders had no role in the design of the study; in the collection, analyses, or interpretation of data; in the writing of the manuscript, or in the decision to publish the results.

\section{References}

1. Naeem, S.B.; Bhatti, R. The Covid-19 'infodemic': A new front for information professionals. Health Inf. Libr. J. 2020, 37, 233-239. [CrossRef] [PubMed]

2. SPIEGEL. Menschen Werfen uns Offenbar vor, wir Hätten die Krankheit ins Dorf Gebracht. Available online: https: / / www.spiegel.de/psychologie/stigma-coronavirus-wie-covid-19-die-beziehung-zu-freunden-und-kollegen-veraendernkann-a-d76839ad-d25b-4063-8f55-884bc86824cf (accessed on 7 September 2020).

3. Heute, Z. Die Angst vor der Wut der Mitmenschen. Available online: https://www.zdf.de/nachrichten/panorama/coronastigma-angst-wut-mitmenschen-100.html (accessed on 27 May 2020).

4. Link, B.G.; Phelan, J.C. Conceptualizing stigma. Annu. Rev. Sociol. 2001, 27, 363-385. [CrossRef]

5. Pescosolido, B.A.; Martin, J.K. The stigma complex. Annu. Rev. Sociol. 2015, 41, 87-116. [CrossRef] 
6. Corrigan, P.W.; Larson, J.E.; Kuwabara, S.A. Social psychology of the stigma of mental illness: Public and self-stigma models. In Social Psychological Foundations of Clinical Psychology; The Guilford Press: New York, NY, USA, 2010.

7. Pryor, J.B.; Reeder, G.D.; Monroe, A.E. The infection of bad company: Stigma by association. J. Personal. Soc. Psychol. 2012, 102, 224. [CrossRef]

8. Berger, B.E.; Ferrans, C.E.; Lashley, F.R. Measuring stigma in people with HIV: Psychometric assessment of the HIV stigma scale. Res. Nurs. Health 2001, 24, 518-529. [CrossRef]

9. Quinn, D.M.; Chaudoir, S.R. Living with a concealable stigmatized identity: The impact of anticipated stigma, centrality, salience, and cultural stigma on psychological distress and health. J. Personal. Soc. Psychol. 2009, 97, 634. [CrossRef]

10. Corrigan, P.W.; Watson, A.C.; Barr, L. The self-stigma of mental illness: Implications for self-esteem and self-efficacy. J. Soc. Clin. Psychol. 2006, 25, 875-884. [CrossRef]

11. van der Sanden, R.L.; Stutterheim, S.E.; Pryor, J.B.; Kok, G.; Bos, A.E. Coping with stigma by association and family burden among family members of people with mental illness. J. Nerv. Ment. Dis. 2014, 202, 710-717. [CrossRef]

12. Einarsen, S.; Hoel, H.; Zapf, D.; Cooper, C. Bullying and Emotional Abuse in the Workplace; Taylor \& Francis London \& New York: London, UK; New York, NY, USA, 2002.

13. Östman, M.; Kjellin, L. Stigma by association: Psychological factors in relatives of people with mental illness. Br. J. Psychiatry 2002, 181, 494-498. [CrossRef] [PubMed]

14. Mooney, S.J.; El-Sayed, A.M. Stigma and the etiology of depression among the obese: An agent-based exploration. Soc. Sci. Med. 2016, 148, 1-7. [CrossRef]

15. Carpiniello, B.; Pinna, F. The reciprocal relationship between suicidality and stigma. Front. Psychiatry 2017, 8, 35. [CrossRef] [PubMed]

16. Schomerus, G.; Angermeyer, M. Stigma and its impact on help-seeking for mental disorders: What do we know? Epidemiol Psychiatr. Sci. 2008, 17, 31-37. [CrossRef] [PubMed]

17. Yebei, V.N.; Fortenberry, J.; Ayuku, D.O. Felt stigma among people living with HIV/AIDS in rural and urban Kenya. Afr. Health Sci. 2008, 8, 97-102. [PubMed]

18. Deitch, E.A.; Barsky, A.; Butz, R.M.; Chan, S.; Brief, A.P.; Bradley, J.C. Subtle yet significant: The existence and impact of everyday racial discrimination in the workplace. Hum. Relat. 2003, 56, 1299-1324. [CrossRef]

19. Weber, J.; Angerer, P.; Müller, A. Individual consequences of age stereotypes on older workers. Z. Für Gerontol. Und Geriatr. 2019, 52, 188-205. [CrossRef]

20. von Kardorff, E. Zur Diskriminierung psychisch kranker Menschen. In Diskriminierung; Springer: Berlin/Heidelberg, Germany, 2010; pp. 279-305.

21. Bartlett, J.E.; Bartlett, M.E. Workplace bullying: An integrative literature review. Adv. Dev. Hum. Resour. 2011, 13, 69-84. [CrossRef]

22. Leymann, H. The content and development of mobbing at work. Eur. J. Work Organ. Psychol. 1996, 5, 165-184. [CrossRef]

23. Zapf, D. Mobbing in Organisationen. Ein Überblick zum Stand der Forschung [Bullying at work. An overview of current research]. Z. Für Arb. Organ. 1999, 43, 1-25.

24. Einarsen, S. The nature and causes of bullying at work. Int. J. Manpow. 1999, 20, 16-27. [CrossRef]

25. Kisely, S.; Warren, N.; McMahon, L.; Dalais, C.; Henry, I.; Siskind, D. Occurrence, prevention, and management of the psychological effects of emerging virus outbreaks on healthcare workers: Rapid review and meta-analysis. BMJ 2020, 369 , m1642. [CrossRef]

26. Serrano-Ripoll, M.J.; Meneses-Echavez, J.F.; Ricci-Cabello, I.; Fraile-Navarro, D.; Fiol-deRoque, M.A.; Pastor-Moreno, G.; Castro, A.; Ruiz-Perez, I.; Zamanillo Campos, R.; Goncalves-Bradley, D.C. Impact of viral epidemic outbreaks on mental health of healthcare workers: A rapid systematic review and meta-analysis. J. Affect. Disord. 2020, 277, 347-357. [CrossRef] [PubMed]

27. Paiano, M.; Jaques, A.E.; Nacamura, P.A.B.; Salci, M.A.; Radovanovic, C.A.T.; Carreira, L. Mental health of healthcare professionals in China during the new coronavirus pandemic: An integrative review. Rev. Bras. De Enferm. 2020, 73, e20200338. [CrossRef]

28. Cabarkapa, S.; Nadjidai, S.E.; Murgier, J.; Ng, C.H. The psychological impact of COVID-19 and other viral epidemics on frontline healthcare workers and ways to address it: A rapid systematic review. Brain Behav. Immun. Health 2020, 8, 100144. [CrossRef]

29. Muhidin, S.; Vizheh, M.; Moghadam, Z.B. Anticipating COVID-19-related stigma in survivors and health-care workers: Lessons from previous infectious diseases outbreaks-An integrative literature review. Psychiatry Clin. Neurosci. 2020. [CrossRef] [PubMed]

30. Banerjee, D.; Vaishnav, M.; Sathyanarayana Rao, T.S.; Raju, M.S.V.K.; Dalal, P.K.; Javed, A.; Saha, G.; Mishra, K.K.; Kumar, V.; Jagiwala, M.P. Impact of the COVID-19 pandemic on psychosocial health and well-being in South-Asian (World Psychiatric Association zone 16) countries: A systematic and advocacy review from the Indian Psychiatric Society. Indian J. Psychiatry 2020, 62, S343-S353. [CrossRef]

31. Tng, X.J.J.; Chew, Q.H.; Sim, K. Psychological sequelae within different populations during the COVID-19 pandemic: A rapid review of extant evidence. Singap. Med. J. 2020. [CrossRef] [PubMed]

32. De Brier, N.; Stroobants, S.; Vandekerckhove, P.; De Buck, E. Factors affecting mental health of health care workers during coronavirus disease outbreaks (SARS, MERS \& COVID-19): A rapid systematic review. PLoS ONE 2020, 15, e0244052.

33. Muller, R.A.E.; Stensland, R.S.Ø.; van de Velde, R.S. The mental health impact of the covid-19 pandemic on healthcare workers, and interventions to help them: A rapid systematic review. Psychiatry Res. 2020, 293, 113441. [CrossRef] 
34. Sritharan, J.; Jegathesan, T.; Vimaleswaran, D.; Sritharan, A. Mental Health Concerns of Frontline Workers During the COVID-19 Pandemic: A Scoping Review. Glob. J. Health Sci. 2020, 12, 89-105. [CrossRef]

35. Taylor, S.; Landry, C.A.; Rachor, G.S.; Paluszek, M.M.; Asmundson, G.J. Fear and avoidance of healthcare workers: An important, under-recognized form of stigmatization during the COVID-19 pandemic. J. Anxiety Disord. 2020, 75, 102289. [CrossRef] [PubMed]

36. Dang, A.K.; Le, X.T.T.; Le, H.T.; Tran, B.X.; Do, T.T.T.; Phan, H.T.B.; Nguyen, T.T.; Pham, Q.T.; Ta, N.T.K.; Nguyen, Q.T. Evidence of COVID-19 impacts on occupations during the first Vietnamese national Lockdown. Ann. Glob. Health 2020, 86, 112. [CrossRef] [PubMed]

37. Chaudhary, F.A.; Ahmad, B.; Ahmad, P.; Khalid, M.D.; Butt, D.Q.; Khan, S.Q. Concerns, perceived impact, and preparedness of oral healthcare workers in their working environment during COVID-19 pandemic. J. Occup. Health 2020, 62, e12168. [CrossRef] [PubMed]

38. Mohindra, R.; Soni, R.K.; Suri, V.; Bhalla, A.; Singh, S.M. The experience of social and emotional distancing among health care providers in the context of COVID-19: A study from North India. J. Hum. Behav. Soc. Environ. 2020, 31, 173-183. [CrossRef]

39. Chew, Q.H.; Chia, F.L.-A.; Ng, W.K.; Lee, W.C.I.; Tan, P.L.L.; Wong, C.S.; Puah, S.H.; Shelat, V.G.; Seah, E.-J.D.; Huey, C.W.T. Perceived Stress, Stigma, Traumatic Stress Levels and Coping Responses amongst Residents in Training across Multiple Specialties during COVID-19 Pandemic-A Longitudinal Study. Int. J. Environ. Res. Public Health 2020, 17, 6572. [CrossRef] [PubMed]

40. Do Duy, C.; Nong, V.M.; Van, A.N.; Thu, T.D.; Do Thu, N.; Quang, T.N. COVID-19 related stigma and its association with mental health of health-care workers after quarantined in Vietnam. Psychiatry Clin. Neurosci. 2020, 74, 566-568. [CrossRef] [PubMed]

41. Zhu, Z.; Xu, S.; Wang, H.; Liu, Z.; Wu, J.; Li, G.; Miao, J.; Zhang, C.; Yang, Y.; Sun, W. COVID-19 in Wuhan: Sociodemographic characteristics and hospital support measures associated with the immediate psychological impact on healthcare workers. EClinicalMedicine 2020, 24, 100443. [CrossRef] [PubMed]

42. Landis, J.R.; Koch, G.G. The Measurement of Observer Agreement For Categorical Data. Biometrics 1977, 33, 159-174. [CrossRef]

43. Ijaz, S.; Verbeek, J.; Seidler, A.; Lindbohm, M.-L.; Ojajärvi, A.; Orsini, N.; Costa, G.; Neuvonen, K. Night-shift work and breast cancer-A systematic review and meta-analysis. Scand. J. Work Environ. Health 2013, 431-447. [CrossRef]

44. Kuijer, P.P.F.; Verbeek, J.H.; Seidler, A.; Ellegast, R.; Hulshof, C.T.; Frings-Dresen, M.H.; Van der Molen, H.F. Work-relatedness of lumbosacral radiculopathy syndrome: Review and dose-response meta-analysis. Neurology 2018, 91, 558-564. [CrossRef]

45. Romero Starke, K.; Kofahl, M.; Freiberg, A.; Schubert, M.; Groß, M.L.; Schmauder, S.; Hegewald, J.; Kämpf, D.; Stranzinger, J.; Nienhaus, A. Are Daycare Workers at a Higher Risk of Parvovirus B19 Infection? A Systematic Review and Meta-Analysis. Int. J. Environ. Res. Public Health 2019, 16, 1392. [CrossRef]

46. Callus, E.; Bassola, B.; Fiolo, V.; Bertoldo, E.G.; Pagliuca, S.; Lusignani, M. Stress Reduction Techniques for Health Care Providers Dealing With Severe Coronavirus Infections (SARS, MERS, and COVID-19): A Rapid Review. Front. Psychol. 2020, $11,3325$. [CrossRef] [PubMed]

47. Joo, J.Y.; Liu, M.F. Nurses' barriers to caring for patients with COVID-19: A qualitative systematic review. Int. Nurs. Rev. 2021. [CrossRef]

48. Rahman, M.; Ahmed, R.; Moitra, M.; Damschroder, L.; Brownson, R.; Chorpita, B.; Idele, P.; Gohar, F.; Huang, K.Y.; Saxena, S. Mental Distress and Human Rights Violations During COVID-19: A Rapid Review of the Evidence Informing Rights, Mental Health Needs, and Public Policy Around Vulnerable Populations. Front. Psychiatry 2020, 11, 603875. [CrossRef]

49. Blake, H.; Bermingham, F.; Johnson, G.; Tabner, A. Mitigating the psychological impact of covid-19 on healthcare workers: A digital learning package. Int. J. Environ. Res. Public Health 2020, 17, 2997. [CrossRef] [PubMed]

50. Chatterjee, S.S.; Bhattacharyya, R.; Bhattacharyya, S.; Gupta, S.; Das, S.; Banerjee, B.B. Attitude, practice, behavior, and mental health impact of COVID-19 on doctors. Indian J. Psychiatry 2020, 62, 257.

51. Juan, Y.; Yuanyuan, C.; Qiuxiang, Y.; Cong, L.; Xiaofeng, L.; Yundong, Z.; Jing, C.; Peifeng, Q.; Yan, L.; Xiaojiao, X.; et al. Psychological distress surveillance and related impact analysis of hospital staff during the COVID-19 epidemic in Chongqing, China. Compr. Psychiatry 2020, 103, 152198. [CrossRef] [PubMed]

52. Kackin, O.; Ciydem, E.; Aci, O.S.; Kutlu, F.Y. Experiences and psychosocial problems of nurses caring for patients diagnosed with COVID-19 in Turkey: A qualitative study. Int. J. Soc. Psychiatry 2020, 20764020942788. [CrossRef]

53. Kalateh Sadati, A.; Zarei, L.; Shahabi, S.; Heydari, S.T.; Taheri, V.; Jiriaei, R.; Ebrahimzade, N.; Lankarani, K.B. Nursing experiences of COVID-19 outbreak in Iran: A qualitative study. Nurs. Open 2021, 8, 72-79. [CrossRef]

54. Abdel Wahed, W.Y.; Hefzy, E.M.; Ahmed, M.I.; Hamed, N.S. Assessment of Knowledge, Attitudes, and Perception of Health Care Workers Regarding COVID-19, A Cross-Sectional Study from Egypt. J. Community Health 2020. [CrossRef]

55. Chew, Q.H.; Wei, K.C.; Vasoo, S.; Sim, K. Psychological and Coping Responses of Health Care Workers Toward Emerging Infectious Disease Outbreaks: A Rapid Review and Practical Implications for the COVID-19 Pandemic. J. Clin. Psychiatry $2020,81$. [CrossRef]

56. Fawaz, M.; Samaha, A. The psychosocial effects of being quarantined following exposure to COVID-19: A qualitative study of Lebanese health care workers. Int. J. Soc. Psychiatry 2020, 66, 560-565. [CrossRef]

57. Dye, T.D.; Alcantara, L.; Siddiqi, S.; Barbosu, M.; Sharma, S.; Panko, T.; Pressman, E. Risk of COVID-19-related bullying, harassment and stigma among healthcare workers: An analytical cross-sectional global study. BMJ Open 2020, 10, e046620. [CrossRef] 
58. Elhadi, M.; Msherghi, A.; Elgzairi, M.; Alhashimi, A.; Bouhuwaish, A.; Biala, M.; Abuelmeda, S.; Khel, S.; Khaled, A.; Alsoufi, A. Burnout Syndrome Among Hospital Healthcare Workers During the COVID-19 Pandemic and Civil War: A Cross-Sectional Study. Front. Psychiatry 2020, 11, 1426. [CrossRef]

59. Elhadi, M.; Msherghi, A.; Elgzairi, M.; Alhashimi, A.; Bouhuwaish, A.; Biala, M.; Abuelmeda, S.; Khel, S.; Khaled, A.; Alsoufi, A.; et al. Psychological status of healthcare workers during the civil war and COVID-19 pandemic: A cross-sectional study. J. Psychosom. Res. 2020, 137, 110221. [CrossRef]

60. Greene, T.; Harju-Seppänen, J.; Adeniji, M.; Steel, C.; Grey, N.; Brewin, C.R.; Bloomfield, M.A.; Billings, J. Predictors of PTSD, depression and anxiety in UK frontline health and social care workers during COVID-19. Eur. J. Psychotraumatol. 2020, 12, 1882781. [CrossRef] [PubMed]

61. Khanal, P.; Devkota, N.; Dahal, M.; Paudel, K.; Joshi, D. Mental health impacts among health workers during COVID-19 in a low resource setting: A cross-sectional survey from Nepal. Glob. Health 2020, 16, 89. [CrossRef] [PubMed]

62. Khanal, P.; Devkota, N.; Dahal, M.; Paudel, K.; Mishra, S.R.; Joshi, D. Corona virus fear among health workers during the early phase of pandemic response in Nepal: A web-based cross-sectional study. bioRxiv 2020. [CrossRef]

63. Monterrosa-Castro, A.; Redondo-Mendoza, V.; Mercado-Lara, M. Psychosocial factors associated with symptoms of generalized anxiety disorder in general practitioners during the COVID-19 pandemic. J. Investig. Med. Off. Publ. Am. Fed. Clin. Res. 2020. [CrossRef]

64. Ramaci, T.; Barattucci, M.; Ledda, C.; Rapisarda, V. Social Stigma during COVID-19 and its impact on HCWs outcomes. Sustainability 2020, 12, 3834. [CrossRef]

65. Sharma, M.; Creutzfeldt, C.J.; Lewis, A.; Patel, P.V.; Hartog, C.; Jannotta, G.E.; Blissitt, P.; Kross, E.K.; Kassebaum, N.; Greer, D.M.; et al. Healthcare professionals' perceptions of critical care resource availability and factors associated with mental well-being during COVID-19: Results from a US survey. Clin. Infect. Dis. Off. Publ. Infect. Dis. Soc. Am. 2020. [CrossRef]

66. Tan, W.; Hao, F.; McIntyre, R.S.; Jiang, L.; Jiang, X.; Zhang, L.; Zhao, X.; Zou, Y.; Hu, Y.; Luo, X. Is returning to work during the COVID-19 pandemic stressful? A study on immediate mental health status and psychoneuroimmunity prevention measures of Chinese workforce. Brain Behav. Immun. 2020, 87, 84-92. [CrossRef] [PubMed]

67. Uvais, N.A.; Aziz, F.; Hafeeq, B. COVID-19-Related Stigma and Perceived Stress among Dialysis Staff; Springer: New York City, NY, USA, 2020

68. Uvais, N.A.; Shihabudheen, P.; Hafi, N.A.B. Perceived Stress and Stigma Among Doctors Working in COVID-19-Designated Hospitals in India. Prim. Care Companion CNS Disord. 2020, 22. [CrossRef]

69. Yadav, K.; Laskar, A.R.; Rasania, S. A study on stigma and apprehensions related to COVID-19 among healthcare professionals in Delhi. Int. J. Community Med. Public Health 2020, 7, 4547. [CrossRef]

70. Said, R.M.; El-Shafei, D.A. Occupational stress, job satisfaction, and intent to leave: Nurses working on front lines during COVID-19 pandemic in Zagazig City, Egypt. Environ. Sci. Pollut. Res. 2021, 28, 8791-8801. [CrossRef]

71. Zandifar, A.; Badrfam, R.; Khonsari, N.M.; Mohammadi, M.R.; Asayesh, H.; Qorbani, M. Prevalence and associated factors of posttraumatic stress symptoms and stigma among health care workers in contact with COVID-19 patients. Iran. J. Psychiatry 2020, 15, 340-350. [CrossRef] [PubMed]

72. Chen, X.; Gao, H.; Zou, Y.; Lin, F. Changes in psychological wellbeing, attitude, and information-seeking behavior among people at the epicenter of the COVID-19 pandemic: A panel survey of residents in Hubei province, China. Epidemiol. Infect. 2020. [CrossRef] [PubMed]

73. Abedi, V.; Olulana, O.; Avula, V.; Chaudhary, D.; Khan, A.; Shahjouei, S.; Li, J.; Zand, R. Racial, Economic, and Health Inequality and COVID-19 Infection in the United States. J. Racial Ethn. Health Disparities 2020. [CrossRef]

74. McMahon, S.A.; Ho, L.S.; Brown, H.; Miller, L.; Ansumana, R.; Kennedy, C.E. Healthcare providers on the frontlines: A qualitative investigation of the social and emotional impact of delivering health services during Sierra Leone's Ebola epidemic. Health Policy Plan. 2016, 31, 1232-1239. [CrossRef]

75. See, L.-C.; Shen, Y.-M.; Chen, C.-L.; Huang, T.-M.; Huang, Y.-H.; Huang, H.-C.; Lin, S.-R. Professional attitude of health care workers toward serving HIV/AIDS patients and drug users: Questionnaire design and evaluation of reliability and validity. AIDS Care 2011, 23, 1448-1455. [CrossRef]

76. Reazee, N.; Mardani-Hamooleh, M.; Seraji, M. Nurses' perception of ethical challenges in caring for patients with COVID-19: A qualitative analysis. J. Med. Ethics Hist. Med. 2020, 13, 23.

77. Rizvi Jafree, S.; ul Momina, A.; Naqi, S.A. Significant other family members and their experiences of COVID-19 in Pakistan: A qualitative study with implications for social policy. Stigma Health 2020. [CrossRef]

78. Feroz, A.S.; Pradhan, N.A.; Ahmed, Z.H.; Shah, M.M.; Asad, N.; Saleem, S.; Siddiqi, S. The Perceptions and Experiences of Health-Care Providers During COVID-19 Pandemic in Karachi, Pakistan: An Exploratory Qualitative Study. Pak. An Explor. Qual. Study (6/22/2020) 2020. Available online: https://papers.ssrn.com/sol3/papers.cfm?abstract_id=3634827 (accessed on 15 April 2021).

79. Crowe, S.; Howard, A.F.; Vanderspank-Wright, B.; Gillis, P.; McLeod, F.; Penner, C.; Haljan, G. The effect of COVID-19 pandemic on the mental health of Canadian critical care nurses providing patient care during the early phase pandemic: A mixed method study. Intensive Crit. Care Nurs. 2020, 63, 102999. [CrossRef]

80. Hien, W.; von Schwarzkopf, H. Corona-Gefährdung im Erleben von Pflegekräften-Eine Explorative Studiemit Hinweisen auf Erweiterte Gesundheitsschutzkonzepte; Berufsgenossenschaft für Gesundheitsdienst und Wohlfahrtspflege: Bremen, Germany; p. 2020. 
81. Bhatt, N.; Bhatt, B.; Gurung, S.; Dahal, S.; Jaishi, A.R.; Neupane, B.; Budhathoki, S.S. Perceptions and experiences of the public regarding the COVID-19 pandemic in Nepal: A qualitative study using phenomenological analysis. BMJ Open 2020, 10, e043312. [CrossRef]

82. Lee, N.; Lee, H.-J. South Korean Nurses' Experiences with Patient Care at a COVID-19-Designated Hospital: Growth after the Frontline Battle against an Infectious Disease Pandemic. Int. J. Environ. Res. Public Health 2020, 17, 9015. [CrossRef]

83. Zolnikov, T.R.; Furio, F. Stigma on first responders during COVID-19. Stigma Health 2020, 5, 375-379. [CrossRef]

84. Jesus, T.S.; Kamalakannan, S.; Bhattacharjya, S.; Bogdanova, Y.; Arango-Lasprilla, J.; Bentley, J.; Gibson, B.E.; Papadimitriou, C. People with disabilities and other forms of vulnerability to the COVID-19 pandemic: Study protocol for a scoping review and thematic analysis. Arch. Rehabil. Res. Clin. Transl. 2020, 2, 100079. [CrossRef] [PubMed]

85. Nochaiwong, S.; Ruengorn, C.; Awiphan, R.; Ruanta, Y.; Boonchieng, W.; Nanta, S.; Kowatcharakul, W.; Pumpaisalchai, W.; Kanjanarat, P.; Mongkhon, P.; et al. Mental health circumstances among health care workers and general public under the pandemic situation of COVID-19 (HOME-COVID-19). Medicine 2020, 99, e20751. [CrossRef] [PubMed]

86. Mongkhon, P.; Ruengorn, C.; Awiphan, R.; Thavorn, K.; Hutton, B.; Wongpakaran, N.; Wongpakaran, T.; Nochaiwong, S. Exposure to COVID-19-Related Information and its Association With Mental Health Problems in Thailand: Nationwide, Cross-sectional Survey Study. J. Med. Internet Res. 2021, 23, e25363. [CrossRef] [PubMed]

87. Ruengorn, C.; Awiphan, R.; Wongpakaran, N.; Wongpakaran, T.; Nochaiwong, S.; Outcomes, H.; Health Outcomes and Mental Health Care Evaluation Survey Research Group. Association of job loss, income loss, and financial burden with adverse mental health outcomes during coronavirus disease 2019 pandemic in Thailand: A nationwide cross-sectional study. Depress. Anxiety 2021, 38, 648-660. [CrossRef]

88. Sulaiman, A.H.; Ahmad Sabki, Z.; Jaafa, M.J.; Francis, B.; Razali, K.A.; Juares Rizal, A.; Mokhtar, N.H.; Juhari, J.A.; Zainal, S.; Ng, C.G. Development of a Remote Psychological First Aid Protocol for Healthcare Workers Following the COVID-19 Pandemic in a University Teaching Hospital, Malaysia. Healthcare 2020, 8, 228. [CrossRef] [PubMed]

89. Newheiser, A.-K.; Barreto, M. Hidden costs of hiding stigma: Ironic interpersonal consequences of concealing a stigmatized identity in social interactions. J. Exp. Soc. Psychol. 2014, 52, 58-70. [CrossRef]

90. Bos, A.E.; Kanner, D.; Muris, P.; Janssen, B.; Mayer, B. Mental illness stigma and disclosure: Consequences of coming out of the closet. Issues Ment. Health Nurs. 2009, 30, 509-513. [CrossRef]

91. Topkaya, N. Gender, Self-Stigma, and Public Stigma in Predicting Attitudes toward Psychological Help-Seeking. Educ. Sci. Theory Pract. 2014, 14, 480-487. [CrossRef]

92. Kulesza, M.; Raguram, R.; Rao, D. Perceived mental health related stigma, gender, and depressive symptom severity in a psychiatric facility in South India. Asian J. Psychiatry 2014, 9, 73-77. [CrossRef] [PubMed]

93. Griffiths, K.M.; Christensen, H.; Jorm, A.F. Predictors of depression stigma. BMC Psychiatry 2008, 8, 1-12. [CrossRef] [PubMed]

94. Kreiner, G.E.; Ashforth, B.E.; Sluss, D.M. Identity dynamics in occupational dirty work: Integrating social identity and system justification perspectives. Organ. Sci. 2006, 17, 619-636. [CrossRef]

95. Mejia, C.; Pittman, R.; Beltramo, J.M.; Horan, K.; Grinley, A.; Shoss, M.K. Stigma \& dirty work: In-group and out-group perceptions of essential service workers during COVID-19. Int. J. Hosp. Manag. 2021, 93, 102772.

96. Hennein, R.; Mew, E.J.; Lowe, S.R. Socio-ecological predictors of mental health outcomes among healthcare workers during the COVID-19 pandemic in the United States. PLOS ONE 2021, 16, e0246602. [CrossRef]

97. Heijnders, M.; Van Der Meij, S. The fight against stigma: An overview of stigma-reduction strategies and interventions. Psychol. Health Med. 2006, 11, 353-363. [CrossRef]

98. International Labour Organization. Managing Work-Related Psychosocial Risks during the COVID-19 Pandemic; ILO: Geneva, Switzerland, 2020.

99. Krendl, A.C.; Pescosolido, B.A. Countries and cultural differences in the stigma of mental illness: The east-west divide. J. Cross-Cult. Psychol. 2020, 51, 149-167. [CrossRef] 